\title{
XXV. On the nebular hypothesis.-IV. Correlations of central force
}

\author{
Pliny Earle Chase LL.D. S.P.A.S.
}

To cite this article: Pliny Earle Chase LL.D. S.P.A.S. (1876) XXV. On the nebular hypothesis.-IV. Correlations of central force, Philosophical Magazine Series 5, 2:10, 198-202, DOI: $10.1080 / 14786447608639090$

To link to this article: http://dx.doi.org/10.1080/14786447608639090

曲 Published online: 13 May 2009.

Submit your article to this journal $\lceil\pi$

ЏII Article views: 2

Q View related articles $\sqsubset$ 
insulates the conducting wire therefrom. In this case, too, we have consequently the conditions of a rapidly charged and discharged condenser, giving rise to reciprocating induced sparks.

13. This conclusion can be directly proved by recurring to the earlier experiments (fig. 2) and introducing across the circuit a wire of suitable resistance to short-circuit the extracurrent so that it may complete its route without having to overleap the break at the key. This is shown in diagram in fig. 6 , where $R$ is a box of resistance-coils introduced between $a$ and $a^{\prime}$. If the wire of which the electromagnet $\mathrm{E}$ is made be stout, its resistance will be a mere trifle, and a resistance of 3 or $4 \mathrm{ohms}$ at $R$ will be sufficient. In some cases a drop of pure water between the points of contact at $K$ has also the result of obviating the extra-current spark. And in every case, when the conditions are so arranged as to obviate the appearance, on breaking contact, of the extra-current spark at $K$, no earth-wire, nor any conductor, will draw a spark from the electromagnet-core-a decisive proof that the sparks so drawn were due to indnction by the statical charge of the accumulating extra-current, and to that alone.

XXV. On the Nebular Hypothesis.-IV. Correlations of Central Force. By Pliny Earle Chase, LL.D., S.P.A.S., Professor of Philosophy in Haverford College.

[Continued from p. 36.]

THE various accordances of planetary mass and position 1 which have been pointed out in preceding papers, the harmonic prediction of a source of planetary disturbance at -269 Earth's radius vector which was confirmed by the sunspot observations of Messrs. De La Rue, Stewart, and Loewy, the comparison of gravitating and chemical force by means of the explosive energy of $\mathrm{H}_{2} \mathrm{O}$, the moasurement of gravitating waves, the agreement between the ratio of selenium-conductivity in sunlight to its conductivity in darkness and the ratio of Sun's light-emitting to its earth-attracting energies, and my various mechanical estimates of solar mass and distance seem to be dependent upon mathematical laws, among which the following merit special consideration.

Let $f=$ velocity communicable, at distance $r$, in time $t$, by any central force varying as $\frac{1}{d^{2}}$,

$f_{1}=$ velocity communicated by a single impulse, or in a single instant, 
Prof. P. E. Chase on the Correlations of Central Force. 199 Then

$f_{n}=n f_{1}=$ velocity communicated by $n$ equal impulses or in $n$ instants, the distance remaing constant.

$\alpha$. If the pressure resulting from $f$ is constant, it must either be exactly counterbalanced by some opposing force or forces, so as to produce relative rest, or the motion must be maintained at a constant distance from the centre, so as to produce circular revolution, or, if the opposing force results from the transformation of interfering revolutions, the two conditions may be combined so as to produce rotation. The velocity of circular revolution $=\sqrt{\overline{r^{2}}}$; the velocity of rotation $=\frac{\sqrt{f r}}{n}$. The value of $n$ may be found by experiment; or if the aggregating medium is homogeneous, it may easily be calculated.

$\beta$. If the pressure is varying, and so exerted as to produce radial motion directly towards the centre, the velocity acquired at any given distance $\rho$ may be found by the equation $v_{\rho}=\left\{\frac{2 f r(r-\rho)}{\rho}\right\}^{\frac{1}{2}}$. At the centre, $\rho=0$ and $v_{\rho}=\infty$.

$\gamma$. If the pressure leads to perpetual radial cscillations synchronous with perpetual circular oscillations under constant pressure, the range of the radial oscillations from the centre is $2 r$, and the mean velocity of radial oscillation is $\frac{2}{\pi} \sqrt{f r}$. The equation $\frac{2}{\pi} \sqrt{f r}=\left\{\frac{f r(2 r-\rho)}{\rho}\right\}^{\frac{1}{2}}$ gives $\rho=\frac{2 \pi^{2} r}{\pi^{2}+4}=1.4232 r$.

$\delta$. The vis viva of the total force varying as $\frac{1}{r}$, the theoretical vis viva of average radial velocity : vis viva of synchronous circular velocity : : $1: 1 \cdot 4232$.

$\epsilon$. By the dynamic theory of gases, the average radial velocity = velocity of constant volume; by the laws of thermodynamics, heat varies as vis viva; and we have already seen $(\alpha)$ that the velocity of circular revolution = velocity of constant pressure. We have thus a mathematical verification of the empirical ratio between heat of constant volume and heat of constant pressure.

$\zeta$. If the constrained synchronous rotation of particles in a spheroid, and the free revolution of exterior particles under the influence of any central force whatever, are due to the same primitive impulses $(\alpha)$, the velocity of those impulses is the limit of velocity towards or from which both motions tend. Let, therefore, $v_{1}=\sqrt{\overrightarrow{f r}}=$ velocity of free revolution, varying as $\sqrt{\frac{1}{r}}, v_{\mu}=\frac{\sqrt{f r}}{n}=$ constrained velocity at same point, or 
velocity of superficial equatorial rotation varying as $\frac{1}{r}$; then $v_{0}=\left[v_{1}\right]=\left[v_{\mu}\right]=n v_{1}=n^{2} v_{1 /}=n \sqrt{f r}=$ limit of tangential velocity, both of revolution and of rotation, under a reduction of spheroidal volume to an equatorial radius $=\frac{r}{n^{2}}$. Under such reduction all the particles in the equatorial planes would have the velocity of free revolution, or of perfect fluidity, provided the sphere were homogeneous.

$\eta$. If contraction were to go still further, the centrifugal force of rotation would give the nucleal particles orbits of increasing ellipticity. Those orbits would finally become linear, leading to complete dissociation when the mean linear velocity $\frac{2}{\pi}\left[v_{I}\right]$ was sufficient to produce a constant motion with the parabolic perifocal velocity $[\sqrt{2 f r}]$. These velocities coincide at $\frac{2 r}{n^{2} \pi^{2}}$ when $\frac{n^{2} \pi^{2}}{2} \times \frac{2}{\pi} \times \frac{\sqrt{f r}}{n}=\frac{n \pi}{\sqrt{2}} \times \sqrt{2 f r}=n \pi \sqrt{f r}$, thus showing that the limiting velocity between complete dissociation and incipient aggregation is $\pi \times$ the limiting velocity between complete aggregation and incipient dissociation $(n \sqrt{\overline{f r}}$; see $\zeta$ ).

$\theta$. Let $t=$ time of describing $\frac{1}{2} r$ in virtual approach to the centre, under the action of a central force $f,=$ time of describing $r$ in circular revolution or motion under constant pressure ; $f r=\sqrt{\overline{f r}} ; \pi t=$ time of free semicircular oscillation; $n \pi t=$ time of constrained semirotation ; $n \pi f t=n \pi \sqrt{ } \overline{f r}=$ velocity acquired in the time of semirotation. Therefore the velocity of any central impulses which are capable of producing cosmical aggregation, free revolution, and nucleal rotation $=$ velocity produced by constant equatorial pressure acting for a half-rotation.

ı. If the central force is solar gravity, the limiting velocity $(n \pi \sqrt{f r})$ of uniform impulses which would account for solar aggregation, solar rotation, and planetary revolution is the velocity of light. For Earth's distance from Sun being $214 \cdot 86$ solar radii, the velocity of light is $(214 \cdot 86 r \div 497 \cdot 825)=\cdot 4316 r$ per sec. If we make this velocity $=n \pi \sqrt{ } \overline{f r}$, and find the value of $\sqrt{f r}=\sqrt{g r}=2 \pi r \times 214 \cdot 86 \div(365 \cdot 2564 \times 86400)=\cdot 00062705 r$ per sec., $n=\cdot 4316 \div \pi \sqrt{\overline{f r}}=219 \cdot 0894$, and $v_{1 /}=\sqrt{\overline{f r}} \div n=$ $0000028622 r$ per sec. The accordance of this value with other estimates $(\mu)$ is as follows, the unit being $r \div 10000000000$ :-

$\begin{array}{cccc}\text { Spörer. } & \text { Nebular. } & \text { Light. } & \text { Schwabe. } \\ 29533 & 29192 & 28622 & 28511 .\end{array}$


Prof. P. E. Chase on the Correlations of Central Force. 201

$\kappa$. In nebular condensation the atmospheric radius varies as $t^{\frac{2}{3}}$, while the nucleal radius $\propto t^{\frac{1}{2}}$. The nucleal radius therefore varies as the $\frac{3}{4}$ power of the atmospheric radius. In ordinary considerations of the nebular hypothesis, planetary aggregation has been regarded as atmospheric under a velocity varying as $\sqrt{\frac{1}{r}}$; but there are many traceable evidences of nucleal influence under a velocity varying as $\frac{1}{r}$. Obermayer's law (Trans. Vienna Acad.; 'Nature', June 1, 1876, p. 119), that the friction-coefficient of permanent gases varies as the $\frac{3}{4}$ power of that of the coercible gases and as the absolute temperature, seems to be a particular case of the nucleal and dissociating influences of central force.

$\lambda$. The formation of the Jovian and Telluric belts, as indicated by the limiting velocity of their chief planets, shows the combined action of nucleal and atmospheric influences. At the equator $g=32_{1} \mathrm{l} \frac{\mathrm{ft}}{\mathrm{ft}}$; the time of $\frac{1}{2}$ rotation $=43200 \mathrm{sec}$; $\therefore n \pi \sqrt{f r}=43200 \times 32 \frac{1}{12} \mathrm{ft} .=262.5 \mathrm{~m}$. per sec. The limiting velocity for Jupiter is nearly, if not precisely the same; but it cannot be so accurately determined on account of greater uncertainty in regard to the elements of calculation. The solar modulus of light at the principal centre of gravity of our system $(\odot 4)$ is 505400 solar radii ; the nucleal formative is $\therefore \sqrt{505400} \times$ the atmospheric velocity; when the nucleal velocity (c) was the velocity of light $(\cdot 4316 r)$, the atmospheric velocity was $\cdot 4316 r \div \sqrt{505400}=\cdot 0006071 r$. If $\cdot 0006071 r$ $=262.5 \mathrm{~m}$., Sun's distance $(214 \cdot 86 r)=92,902,000$ miles.

$\mu$. Earth was the seat of theoretical primitive nucleal rupture, the velocity of primitive rotation within the nucleus varying as $r$, and communicating a similarly varying vis viva of superficial rotation to the shrinking nucleus. This gives a present theoretical time of solar rotation $=365.256$ days $\div \sqrt{214 \cdot 86}=24.912$ days. The estimates from observation vary between Spörer's (24.624 days) and Schwabe's (25.507 days).

v. According to the same data, the present height of possible solar atmosphere, or the radius of equal centripetal and centrifugal solar force, should be $(214 \cdot 86)^{\frac{2}{3}} r=35 \cdot 873 r$; the time of planetary revolution at Sun 31558150 sec. $\div(214 \cdot 86)^{\frac{\pi}{2}}=10020 \cdot 242$ sec.; the present limit of uniform planetary velocity $2 \pi r$ $\div 10020 \cdot 242=00062705 \mathrm{r}$ per sec.; the present solar modulus of light $(\cdot 4316 \div 00062705)^{2} r=473755 \cdot 65 r$; the present modulus-atmosphere $35 \cdot 873 \times$ modulus $=16995141 r$. 
202 Prof. P. E. Chase on the Correlations of Central Force.

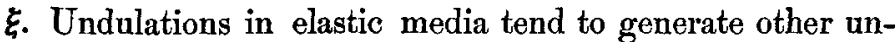
dulations in arithmetic, geometric, harmonic, and various figurate progressions.

o. Radial oscillations through $\rho=m r$ give the central dissociating velocity $\sqrt{2 f r}$ at $\frac{m r}{m+1}$.

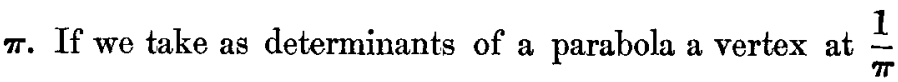
solar radius, or the primitive circumference of complete association $(\eta)$, and an abscissa at present surface of modular atmosphere $(v) \chi_{35 \cdot 373}=35.873 \times$ modulus, the general equation $\chi_{n}=\xi \eta \pm n \zeta^{n^{2}}$ furnishes 27 abscissas in regular progression, of which 9 are between Sun and Mercury, 9 are between Neptune and $\alpha$ Centauri, and the central 9 represent points of dissociating nebular velocity (o), as will be seen by the following Table :-

\begin{tabular}{|c|c|c|c|c|c|}
\hline$n$. & $\log \chi_{n}$. & Log sec. per. & Log mean. & Log sec. aph. & $m r \div(m+1)$ \\
\hline $\begin{array}{r}9 \cdot 87322 \\
10 \cdot 87322 \\
11 \cdot 87322 \\
12 \cdot 87322 \\
13 \cdot 87322 \\
14 \cdot 87322 \\
15 \cdot 87322 \\
16 \cdot 87322 \\
17 \cdot 87322 \\
36 \cdot 87322\end{array}$ & $\begin{array}{l}1 \cdot 6774 \\
1.9099 \\
2 \cdot 1541 \\
2 \cdot 4102 \\
2 \cdot 6779 \\
2 \cdot 9575 \\
3 \cdot 2488 \\
3.5519 \\
3 \cdot 8668 \\
7 \cdot 6622\end{array}$ & $\begin{array}{c}1 \cdot 6806 \\
1 \cdot 8586 \\
2 \cdot 1256 \\
2 \cdot 3248 \\
\ldots \ldots . . \\
2.9420 \\
3 \cdot 2064 \\
3 \cdot 5217 \\
3 \cdot 8614 \\
7 \cdot 6571\end{array}$ & $\begin{array}{c}1 \cdot 7950 \\
1 \cdot 8905 \\
2 \cdot 1561 \\
2 \cdot 3901 \\
\ldots \ldots \ldots \\
2.96 \% 2 \\
3 \cdot 2447 \\
3 \cdot 5571 \\
3 \cdot 8678 \\
\ldots \ldots \ldots\end{array}$ & $\begin{array}{l}1 \cdot 8855 \\
\mathbf{1} \cdot 9200 \\
2 \cdot 1845 \\
2 \cdot 4468 \\
\ldots \ldots . \\
2 \cdot 9949 \\
3 \cdot 2799 \\
3 \cdot 5897 \\
3.8740 \\
7 \cdot 6860\end{array}$ & 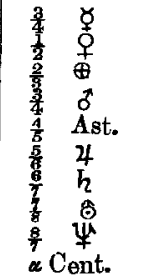 \\
\hline
\end{tabular}

The tabular logarithms for a Centauri $(7 \cdot 6571$ and $7 \cdot 6860)$ represent respectively the least and greatest estimates that have been made of its distance. The planetary secular perihelia and aphelia are the values computed by Stockwell (Smithsonian Contributions, 232, pp. 37, 38).

$\rho$. Weber, Kohlrausch, and Maxwell have identified the velocity which connects electrostatic with electrodynamic action with the velocity of light. These accordances extend the mathematical correlation to heat ( $\alpha$ to $\epsilon$ inclusive), cosmical and molecular aggregation, dissociation, rotation, and revolution ( $\alpha$ to $\theta$ inclusive), gravitation ( $\iota$ to $\pi$ inclusive), and all central forces ( $\alpha$ to $\delta$ and $\xi$ to $\theta$ inclusive, $\xi, a$ ). 